\title{
Azimuthal correlations of high transverse momentum jets at next-to-leading order in the parton branching method
} \author{
Q. Wang ${ }^{3,19}$, H. Yang ${ }^{3,19}$, Y. Zhou ${ }^{20}$ \\ ${ }^{1}$ Department of Physics, Tanta University, Tanta, Egypt \\ ${ }^{2}$ Faculty of Science, Center for High Energy Physics (CHEP-FU), Fayoum University, Faiyum, Egypt \\ ${ }^{3}$ Deutsches Elektronen-Synchrotron DESY, Hamburg, Germany \\ ${ }^{4}$ Università degli Studi Milano-Bicocca, Milan, Italy \\ ${ }^{5}$ Interuniversity Institute for High Energies (IIHE), Université libre de Bruxelles, Brussels, Belgium \\ ${ }^{6}$ Faculty of Natural Sciences and Mathematics, University of Montenegro, Podgorica, Montenegro \\ ${ }^{7}$ Ruđer Bošković Institute, Zagreb, Croatia \\ ${ }^{8}$ National Institute for Nuclear Physics INFN and University of Bari, Bari, Italy \\ ${ }^{9}$ Adiyaman University, Adiyaman, Turkey \\ ${ }^{10}$ Moscow State University, Moscow, Russia \\ ${ }^{11}$ Elementary Particle Physics, University of Antwerp, Antwerp, Belgium \\ ${ }^{12}$ University of Oxford, Oxford, UK \\ 13 Universidad Autónoma de Madrid, Madrid, Spain \\ ${ }^{14}$ Universidade Federal de Pelotas, Pelotas, Brazil \\ 15 University of Edinburgh, Edinburgh, UK \\ ${ }^{16}$ Department of Astronomy and Theoretical Physics, Lund University, Lund, Sweden \\ ${ }^{17}$ Department of Physics, Ihsan Dogramaci Bilkent University, Ankara, Turkey \\ 18 Max-Planck Institut für Physik, Munich, Germany \\ 19 School of Physics, Peking University, Beijing, China \\ ${ }^{20}$ University of Cambridge, Cambridge, UK
}

M. I. Abdulhamid ${ }^{1}$ ， M. A. Al-Mashad ${ }^{2}$ ，A. Bermudez Martinez ${ }^{3}$ ，G. Bonomelli ${ }^{4}$ ， I. Bubanja ${ }^{5,6}$, N. Crnković ${ }^{7}$ F. Colombina ${ }^{3}$ ， B. D'Anzi ${ }^{8}$ ，S. Cerci ${ }^{9}$ ，M. Davydov ${ }^{10}$ ， L. I. Estevez Banos ${ }^{3}$ ， N. Forzano ${ }^{4}$ ，F. Hautmann ${ }^{11,12}$, H. Jung ${ }^{3, a}{ }_{\mathbb{D}}$, S. Kim ${ }^{12}$, A. León Quirós ${ }^{13}$, D. E. Martins ${ }^{14}$, M. Mendizabal ${ }^{3}$, K. Moral Figueroa ${ }^{15}$, S. Prestel ${ }^{16}$, S. Taheri Monfared ${ }^{3}$ ，C. Süslï ${ }^{17}$ ，D. Sunar Cerci $^{9}$ ，A. M. van Kampen ${ }^{11}$ ，P. Van Mechelen ${ }^{11}$ ，A. Verbytskyi ${ }^{18}$,

Received: 22 December 2021 / Accepted: 4 January 2022 / Published online: 13 January 2022

(C) The Author(s) 2022

\begin{abstract}
The azimuthal correlation, $\Delta \phi_{12}$, of high transverse momentum jets in pp collisions at $\sqrt{s}=13 \mathrm{TeV}$ is studied by applying PB-TMD distributions to NLO calculations via MCatNLO together with the PB-TMD parton shower. A very good description of the cross section as a function of $\Delta \phi_{12}$ is observed. In the back-to-back region of $\Delta \phi_{12} \rightarrow \pi$, a very good agreement is observed with the PB-TMD Set 2 distributions while significant deviations are obtained with the PB-TMD Set 1 distributions. Set 1 uses the evolution scale while Set 2 uses transverse momentum as an argument in $\alpha_{\mathrm{s}}$, and the above observation therefore confirms the importance of an appropriate soft-gluon coupling in angular ordered parton evolution. The total uncertainties of the predictions are dominated by the scale uncertainties of the matrix element, while the uncertainties coming from the PB-TMDs and the corresponding PB-TMD shower are very small. The $\Delta \phi_{12}$ measurements are also compared with
\end{abstract}

a e-mail: hannes.jung@desy.de (corresponding author) predictions using MCatNLO together PYTHIA8, illustrating the importance of details of the parton shower evolution.

\section{Introduction}

The description of the cross section of high $p_{\mathrm{T}}$ jets in protonproton (pp) collisions is one of the most important tests of predictions obtained in Quantum Chromodynamics (QCD), and much progress has been achieved in the description of inclusive jets [1-12] by applying next-to-leading (NLO) [13$16]$ and next-to-next-to-leading-order (NNLO) calculations [17-20]. In multijet production, the azimuthal angle $\Delta \phi_{12}$ between the two highest transverse momentum $p_{\mathrm{T}}$-jets is an inclusive measurement of additional jet radiation. At leading order (LO) in strong coupling $\alpha_{\mathrm{s}}$, where only two jets are present, the jets are produced back-to-back, with $\Delta \phi_{12}=\pi$, while a deviation from this back-to-back configuration indi- 
cates the presence of additional jets, and only higher-order calculations can describe the observations. The azimuthal correlation between two jets has been measured in p $\bar{p}$ collisions at a center-of-mass energy of $\sqrt{s}=1.96 \mathrm{TeV}$ by the $\mathrm{D} 0$ collaboration $[21,22]$ and in pp collisions by the ATLAS Collaboration at $\sqrt{s}=7 \mathrm{TeV}$ [23] and by the CMS Collaboration at $\sqrt{s}=7,8$, and $13 \mathrm{TeV}$ [24-27]. When measurements of azimuthal correlations of dijets are compared with LO or NLO computations supplemented by parton showers, deviations of $50 \%$ are observed in the medium $\Delta \phi_{12}$ region even at NLO (see e.g. $[25,26]$ ), which requires a more detailed understanding. In the $\Delta \phi_{12} \rightarrow \pi$ region, deviations of up to $10 \%$ are observed [27], significantly larger than the experimental uncertainties.

Since initial state parton radiation moves the jets away from the $\Delta \phi_{12}=\pi$ region, it is appropriate to investigate the implications of transverse momentum dependent parton densities (TMDs [28]) in the description of the $\Delta \phi_{12}$ measurements. Kinematic effects of the initial-state transverse momenta in the interpretation of jet measurements were pointed out in [29,30]. The region $\Delta \phi_{12} \rightarrow \pi$ is especially sensitive to soft multi-gluon emissions, for which QCD resummation is needed. Calculations at leadinglogarithm have been obtained in Ref. [31]. A calculation based on TMD distributions is found in Refs. [32,33] and further investigated in $[34,35]$. However, in the region $\Delta \phi_{12} \rightarrow \pi$ soft-gluon effects are expected which lead to socalled factorization-breaking [36-38]. An indirect strategy to explore the potential impact of these effects is to compare calculations which assume factorization with high-precision measurements.

The Parton Branching (PB)-method [39,40] allows one to determine TMD parton distributions. With these PB-TMD distributions a very good description of the Drell-Yan process [41] is achieved at the LHC [42] as well as at lower energies [43]. Drell-Yan lepton pair production in association with jet final states is also well described using the TMD jet merging [44]. In Ref. [45] it is shown that $\mathrm{Z}+\mathrm{b}$ production is also well described. TMD parton distributions have been used together with off-shell matrix elements at the lowest order in Refs. [46-48] showing a reasonably good description of the measurements.

In this article we investigate in detail high- $p_{\mathrm{T}}$ dijet production by applying the PB formulation of TMD evolution together with NLO calculations of the hard scattering process in the MADGRAPH5_AMC@NLO [49] framework. We first give a very brief recap of the PB distributions in Sect. 2. In Sect. 3 we describe how TMDs and TMD parton showers are included in the Monte Carlo generator CASCADE3 [50]. We discuss predictions obtained by applying fixed-order NLO perturbative calculations and study the region where soft gluon resummation becomes important. We show predictions using PB-TMDs together with TMD parton shower in Sect. 4 .
We compare these predictions with the one using the PYTHIA8 parton shower. We finally give conclusions in Sect. 5.

\section{PB TMDs}

The PB method $[39,40]$ provides a solution of evolution equations for collinear and TMD parton distributions. The equations are solved by applying the concept of resolvable and non-resolvable branchings with Sudakov form factors providing the probability to evolve from one scale to another without resolvable branching. The method is described in Refs. [40,51].

For the numerical calculations we use the NLO parton distribution sets, PB-NLO-2018-Set 1 and PB-NLO2018-Set 2, as obtained in Ref. [51] from a fit to inclusive deep inelastic scattering precision measurements at HERA [52]. Both the collinear and TMD distributions are available in TMDLiB $[53,54]$, including uncertainty bands. PBNLO-2018-Set 1 corresponds at collinear level to HERAPDF 2.0 NLO [52], while PB-NLO-2018-Set 2 uses transverse momentum (instead of the evolution scale in Set1) for the scale in the running coupling $\alpha_{\mathrm{s}}$ which corresponds to the angular ordering of soft gluon emissions in the initial-state parton evolution [55-58].

In Fig. 1 the distributions of the collinear densities from Set 1 and Set 2 are shown for up-quarks at evolution scales of $\mu=100$ and $1000 \mathrm{GeV}$, typical for multi-jet production described below. The collinear densities are also available in a format compatible with LHAPDF [59], and can be used in calculations of physical processes at NLO. In Fig. 2 we show the TMD distributions for up-quarks at $x=0.01$ and $\mu=10$ and $100 \mathrm{GeV}$. The differences between Set 1 and Set 2 are clearly visible in the small $k_{\mathrm{T}}$-region.

The uncertainties of the TMD distributions include both experimental and model uncertainties, as determined in Ref. [51]. In general, it is observed that those uncertainties are small; for $k_{\mathrm{T}}>1 \mathrm{GeV}$ they are of the order of $2-3 \%$.

\section{Multijet production}

The predictions for multijet production at NLO are obtained using the MADGRAPH5_AMC@NLO [49] framework. We used MADGRAPH5_AMC@NLO in two different modes: one is the fixed NLO mode, in which only partonic events are produced, without parton shower and hadronization, and the other one is the real MC@NLO mode, in which infrared subtraction terms are included to avoid double counting of parton emissions between matrix-element and parton-shower calculations, so that events need to be supplemented with a parton shower (or with PB TMD evolution) in order to produce a physical cross section. 

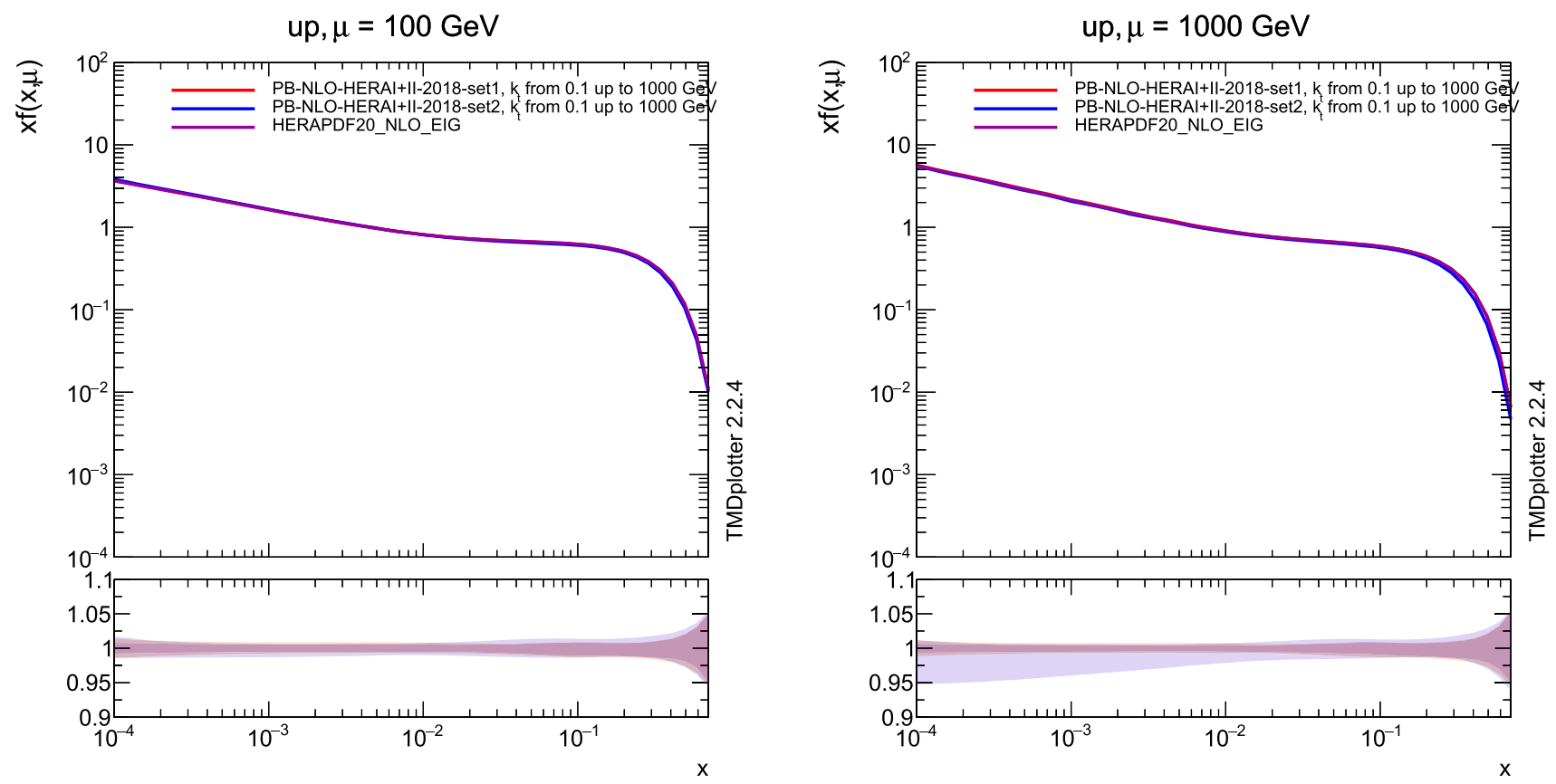

Fig. 1 Collinear parton density distributions for up quarks (PB-NLO-2018-Set 1, PB-NLO-2018-Set 2 and HERAPDF2.0) as a function of $x$ at $\mu=100$ and $1000 \mathrm{GeV}$. In the lower panel the uncertainties are shown

Fixed NLO dijet production is calculated within the MADGRAPH5_AMC@NLO framework. Technically, in the fixed NLO mode, MADGRAPH5_AMC@NLO (version 2.9.3) produces event files with the partonic configuration in LHE format [60] which can be processed through CASCADE3 [50] combining events and counter events (due to infrared subtraction) so that they are treated as one event for a proper calculation of statistical uncertainties. In the fixed NLO mode, the MADGRAPH5_AMC@NLO event record is kept without any modification. Processing through CASCADE3 has a significant advantage that a fixed NLO calculation can be obtained making use of all the analyses coded in Rivet [61].

In the MC@NLO mode, subtraction terms are included which depend on the parton shower used. For the PB-TMDs and the PB-TMD parton shower we use HERwIG6 [62,63] subtraction terms, as already applied in Z and Drell-Yan analyses [42,43], motivated by the angular ordering in the PB evolution. MADGRAPH5_AMC@NLO (version 2.6.4, hereafter labeled MCatNLO) [49] together with the NLO PB parton distributions with $\alpha_{\mathrm{s}}\left(M_{\mathrm{Z}}\right)=0.118$ is used for NLO calculation of dijet production. The matching scale $\mu_{m}$, which limits the contribution from PB-TMDs and TMD showers ( $\mu_{m}=$ SCALUP included in the LHE file), guarantees that the overlap with real emissions from the matrix element is minimized according to the subtraction of counterterms in the MC@NLO method. The factorization and renormalization scale in MCatNLO is set to $\mu_{R, F}=\frac{1}{2} \sum_{i} \sqrt{m_{i}^{2}+p_{t, i}^{2}}$, where the index $i$ runs over all particles in the matrix element final state. This scale is also used in the PB-TMD parton distribution $\mathcal{A}\left(x, k_{\mathrm{T}}, \mu\right)$.

In CASCADE3, as described in detail in Ref. [50], the transverse momentum of the initial state partons is calculated according to the distribution of $k_{\mathrm{T}}$ provided by the PB-TMD $\mathcal{A}\left(x, k_{\mathrm{T}}, \mu\right)$ at given longitudinal-momentum fraction $x$ and evolution scale $\mu$. This transverse momentum is used for the initial state partons provided by MCatNLO, and their longitudinal momentum is adjusted such that the mass and the rapidity of the dijet system is conserved, similar to what has been done in the Drell-Yan case [43]. The initial state TMD parton shower is included in a backward evolution scheme, respecting all parameters and constraints from the PB-TMD. The kinematics of the hard process are not changed by the shower, after the $k_{\mathrm{T}}$ from the TMD is included. The final state parton shower is obtained with the corresponding method implemented in PYTHIA6 [64], by vetoing emissions which do not satisfy angular ordering (MSTJ $(42)=2$ ).

In Fig. 3 we show results for the transverse momentum distribution of the dijet system $p_{\mathrm{T}, 12}$ and the azimuthal correlation $\Delta \phi_{12}$ between the two leading jets as obtained from the MCatNLO calculation at fixed NLO (blue curve), at the level including subtraction terms (LHE level, green curve) and after inclusion of PB-TMDs (red curve). One can clearly observe the rising cross section of the fixed NLO calculation towards small $p_{\mathrm{T}, 12}$ (or at large $\Delta \phi_{12}$ ). This is the region in $p_{\mathrm{T}, 12}$ and $\Delta \phi_{12}$ where the subtraction terms are relevant and a physical prediction is obtained when PB-TMDs and parton showers are included. The jets are defined with the 

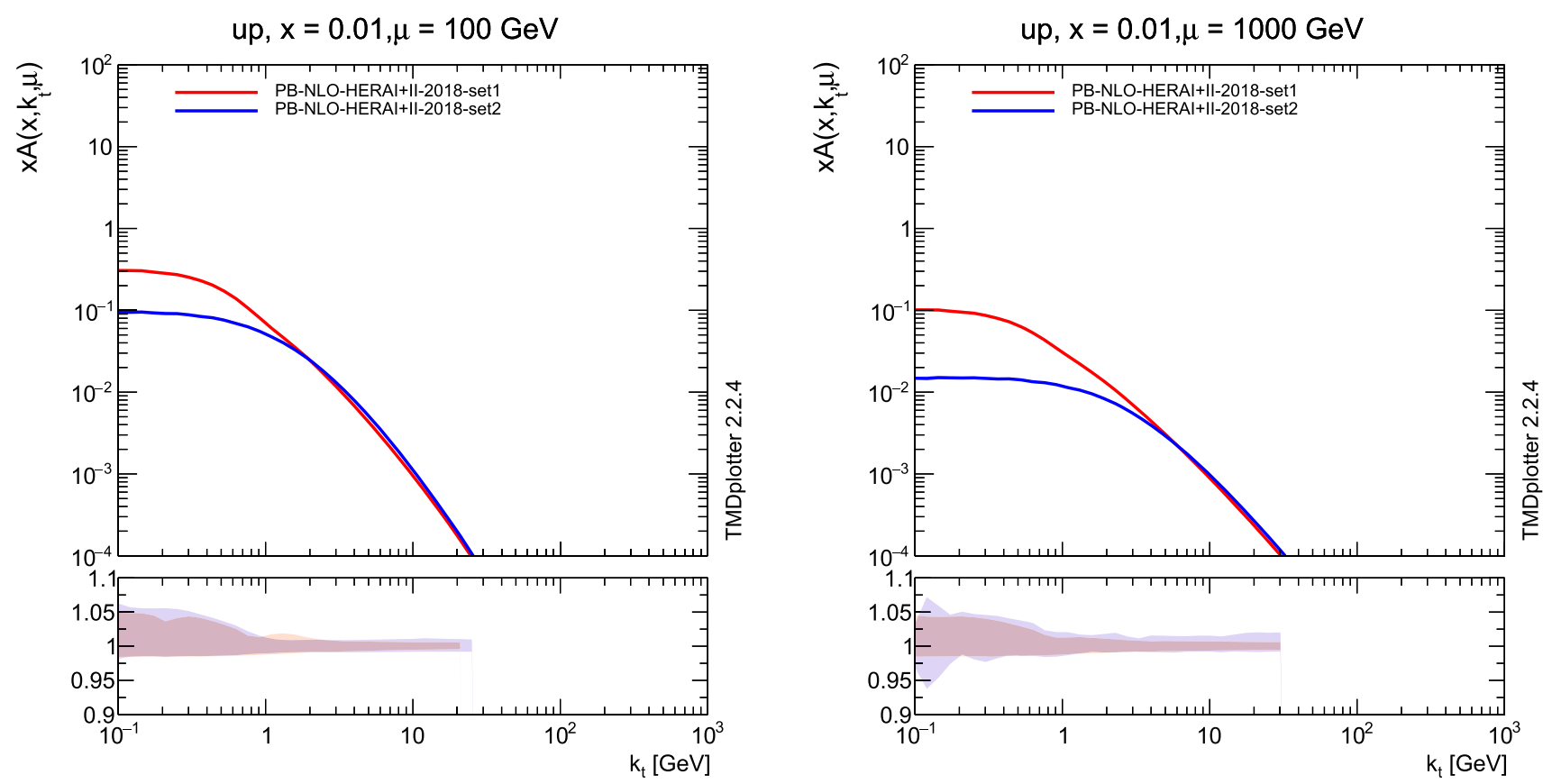

Fig. 2 TMD parton density distributions for up quarks (PB-NLO-2018-Set 1 and PB-NLO-2018-Set 2) as a function of $k_{\mathrm{T}}$ at $\mu=100$ and $1000 \mathrm{GeV}$ and $x=0.01$. In the lower panels show the full uncertainty of the TMDs, as obtained from the fits [51]
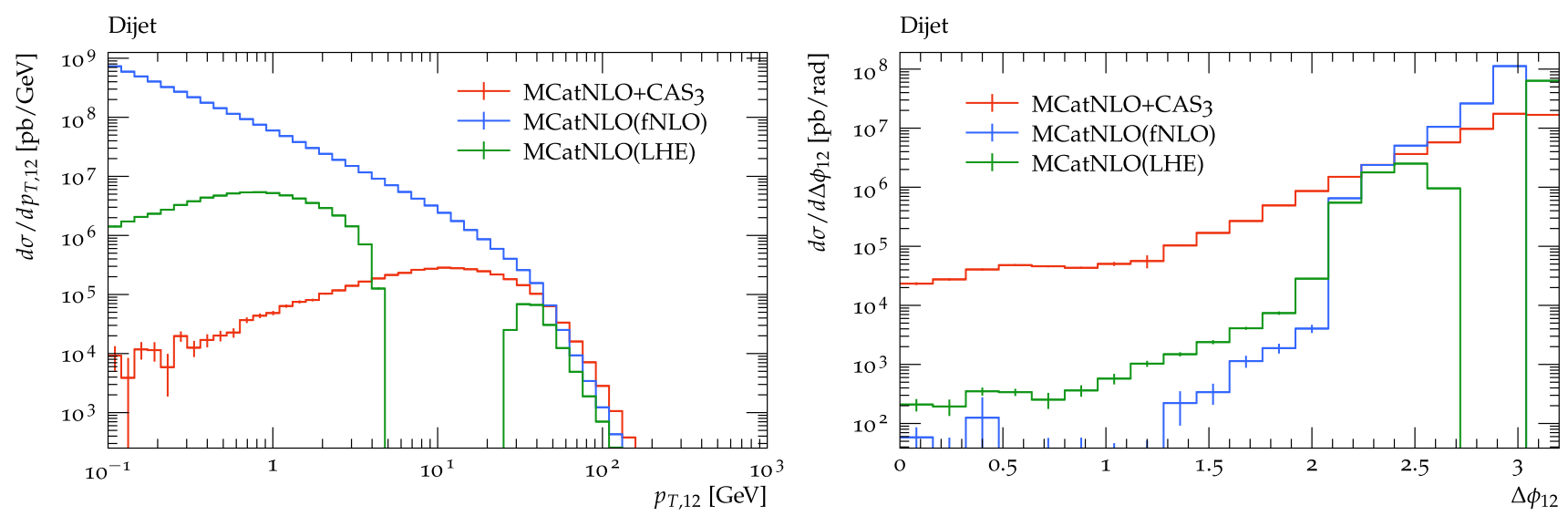

Fig. 3 Transverse momentum spectrum of the dijet system $p_{\mathrm{T}, 12}$ (left) and $\Delta \phi_{12}$ distribution (right). The predictions are shown for fixed NLO (MCatNLO(fNLO), the (unphysical) LHE level (MCatNLO(LHE)) and after inclusion of PB-TMDs (MCatNLO+CAS3)

anti- $k_{\mathrm{T}}$ jet-algorithm [65], as implemented in the FASTJET package [66], with a distance parameter of $\mathrm{R}=0.4$ and a transverse momentum $p_{\mathrm{T}}>200 \mathrm{GeV}$. The use of jets (instead of partons) is the reason for the tail towards small $\Delta \phi_{12}$ in the MCatNLO(LHE) and MCatNLO(fNLO) calculation.

\section{Azimuthal correlations in multijet production}

We next apply the framework described in the previous section, based on the matching of PB-TMDs with NLO matrix elements, to describe the measurement of azimuthal correlations $\Delta \phi_{12}$ obtained by CMS at $\sqrt{s}=13 \mathrm{TeV}$ [26] and in the back-to-back region $\left(\Delta \phi_{12} \rightarrow \pi\right)$ [27]. Only leading jets with a transverse momentum of $p_{\mathrm{T}}^{\text {leading }}>$ $200 \mathrm{GeV}$ are considered. We show distributions of $\Delta \phi_{12}$ for $p_{\mathrm{T}}^{\text {leading }}>200 \mathrm{GeV}$ as well as for the very high $p_{\mathrm{T}}$ region of $p_{\mathrm{T}}^{\text {leading }}>1000 \mathrm{GeV}$, where the jets appear very collimated. We apply the collinear and TMD set PB-NLO2018-Set 2, unless explicitly specified, with running coupling $\alpha_{\mathrm{s}}\left(m_{\mathrm{Z}}\right)=0.118$. We may estimate the theoretical uncertainties on the predictions by considering two kinds of uncertainties: those that come from variation of the arbitrary scales that appear in the various factors that enter the jet cross section, and those that come from the determination of the TMD parton distributions and showers. The former include 



Fig. 4 Azimuthal correlation $\Delta \phi_{12}$ for $p_{\mathrm{T}}^{\text {leading }}>200 \mathrm{GeV}$ (left) and $p_{\mathrm{T}}^{\text {leading }}>1000 \mathrm{GeV}$ (right) as measured by CMS [26] compared with predictions from MCatNLO+CAS3. Shown are the uncertainties coming from the scale variation (as described in the text) as well as the uncertainties coming from the TMD

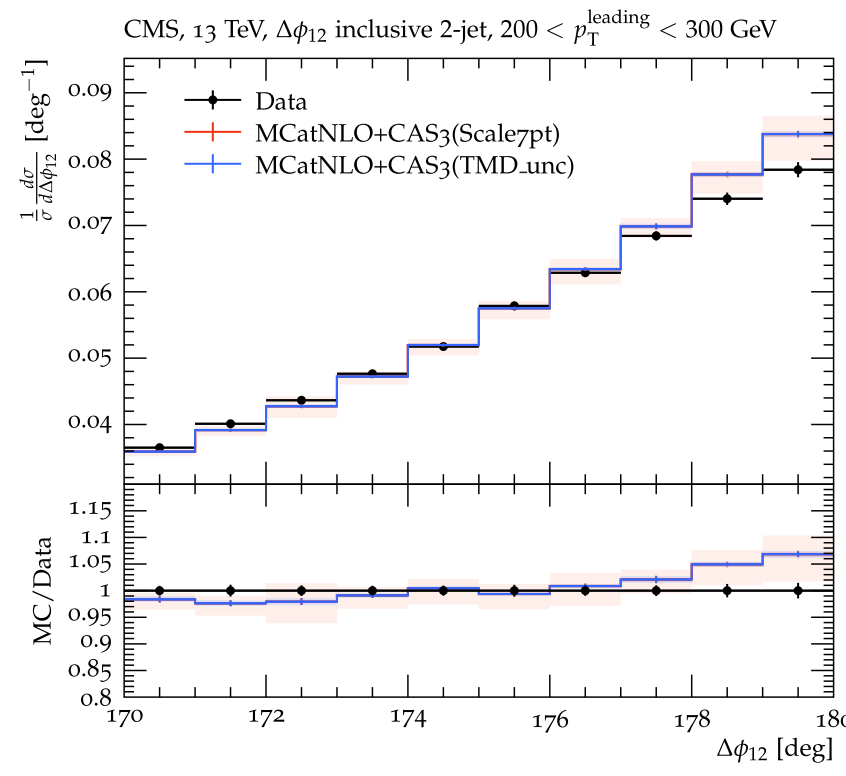

Fig. 5 Azimuthal correlation $\Delta \phi_{12}$ in the back-to-back region for $p_{\mathrm{T}}^{\text {leading }}>200 \mathrm{GeV}$ (left) and $p_{\mathrm{T}}^{\text {leading }}>1000 \mathrm{GeV}$ (right) as measured by CMS [27] compared with predictions from MCatNLO+CAS3.

the renormalization scale in the strong coupling, the factorization scale used in the parton distribution and the matching scale to combine the matrix element and PB TMD. The latter include both experimental and model uncertainties in the TMD extraction. As regards the scale variations, we present results corresponding to the 7-point scheme variation around the central values for the renormalization and factorization

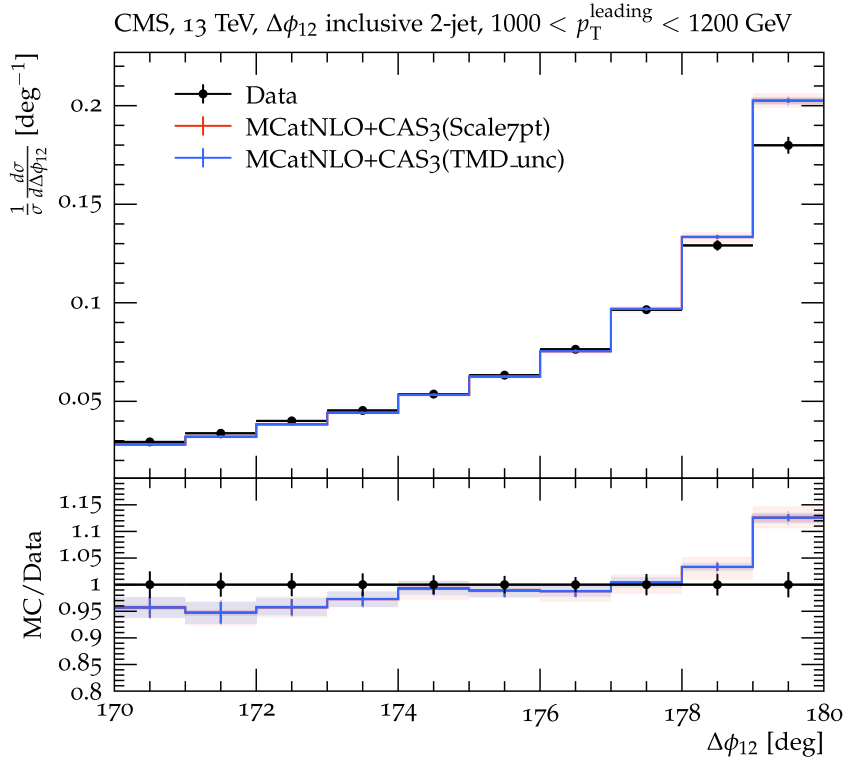

Shown are the uncertainties coming from the scale variation (as described in the text) as well as the uncertainties coming from the TMD

scale (avoiding the extreme cases of variation). We have studied the dependence on the matching scale $\mu_{m}$ and found that is within the band of variation of factorization and renormalization scales. The experimental and model uncertainties on the determination of the TMD distributions as described in [51] are included. 

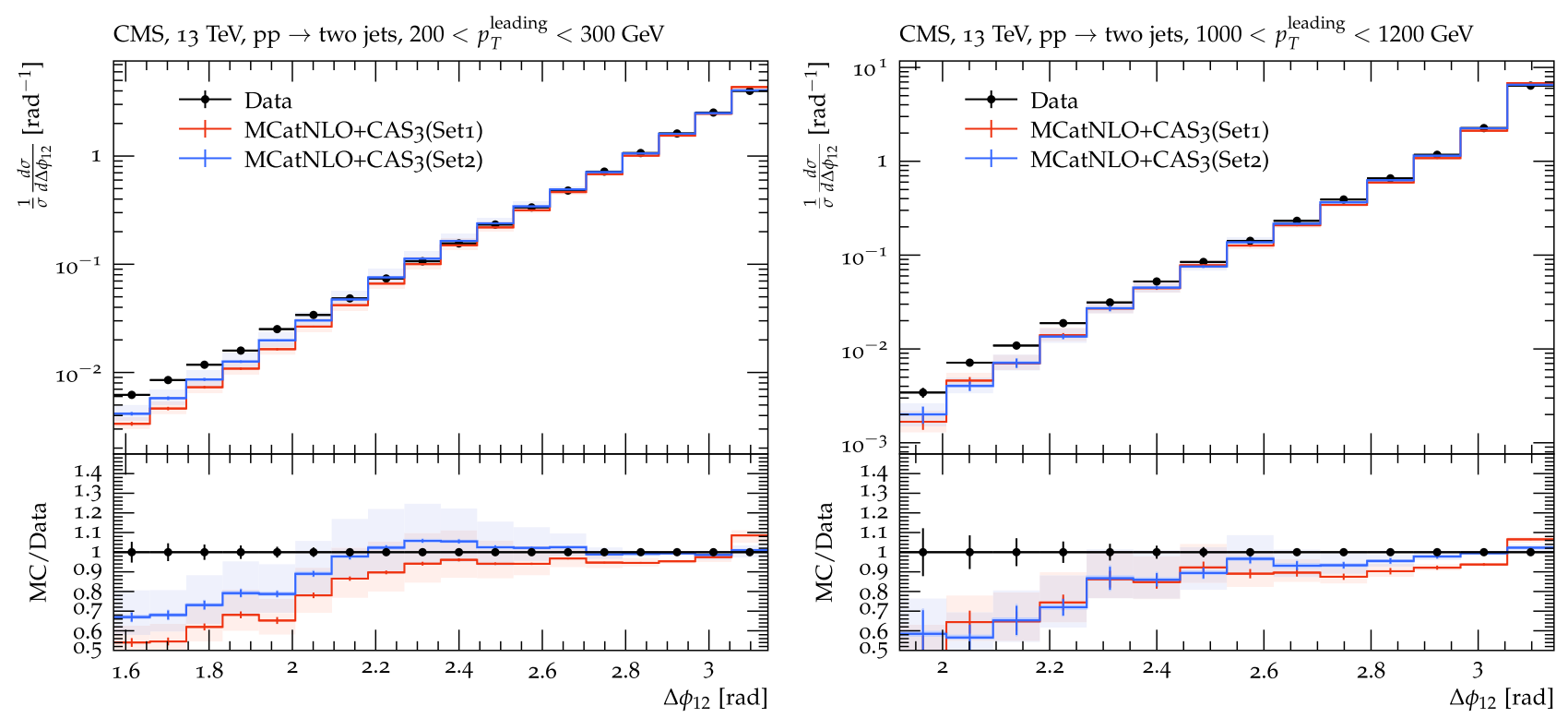

Fig. 6 Azimuthal correlation $\Delta \phi_{12}$ for $p_{\mathrm{T}}^{\text {leading }}>200 \mathrm{GeV}$ (left) and $p_{\mathrm{T}}^{\text {leading }}>1000 \mathrm{GeV}$ (right) as measured by CMS [26] compared with predictions from MCatNLO+CAS3. Shown are the uncertainties coming from the scale variation (as described in the text) as well as the uncertainties coming from the TMD

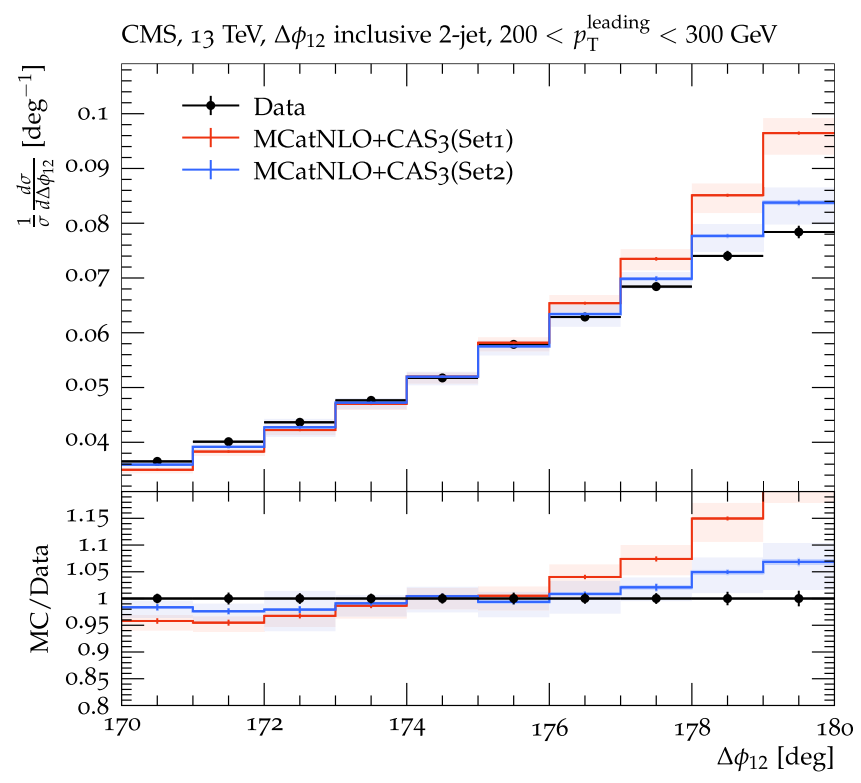

Fig. 7 Azimuthal correlation $\Delta \phi_{12}$ in the back-to-back region for $p_{\mathrm{T}}^{\text {leading }}>200 \mathrm{GeV}$ (left) and $p_{\mathrm{T}}^{\text {leading }}>1000 \mathrm{GeV}$ (right) as measured by CMS [27] compared with predictions from MCatNLO+CAS3.

In Fig. 4 we show a comparison of the measurement by CMS [26] for different values of $p_{\mathrm{T}}^{\text {leading }}$ with the calculation MCatNLO+CAS3 including PB-TMDs, parton shower, and hadronization. The uncertainties from scale variation and TMD determination are shown separately.

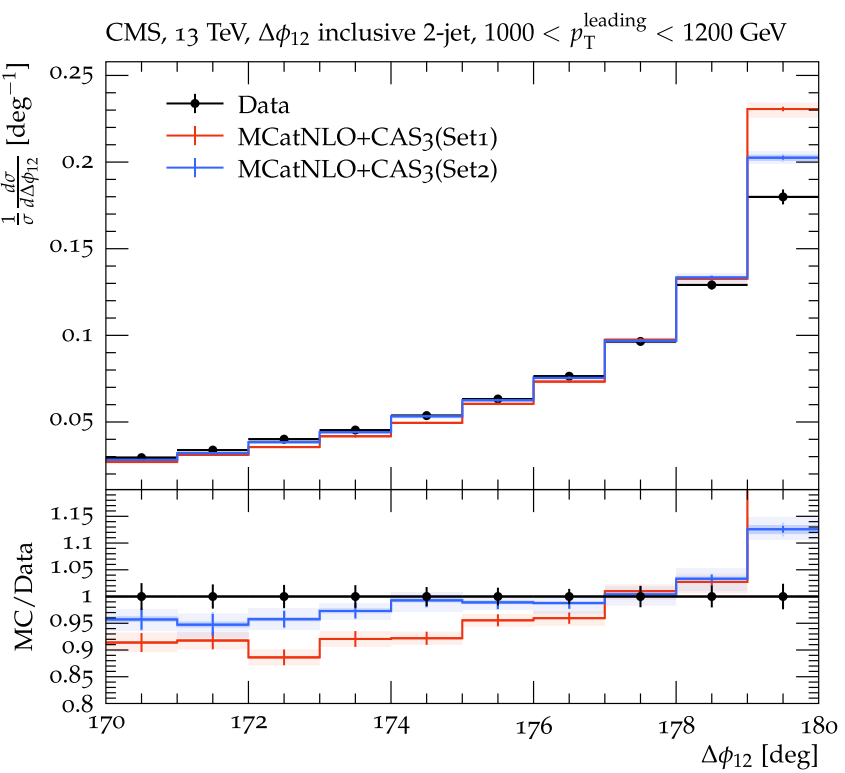

Shown are the uncertainties coming from the scale variation (as described in the text) as well as the uncertainties coming from the TMD

In Fig. 5 the measured $\Delta \phi_{12}$ distribution [27] in the backto-back region is compared with the prediction MCatNLO + CAS3.

In general, the measurements are very well described, especially in the back-to-back region. The scale uncertainty is significantly larger than the TMD uncertainty, especially in the low $p_{\mathrm{T}}^{\text {leading }}$ region. A difference between the measure- 


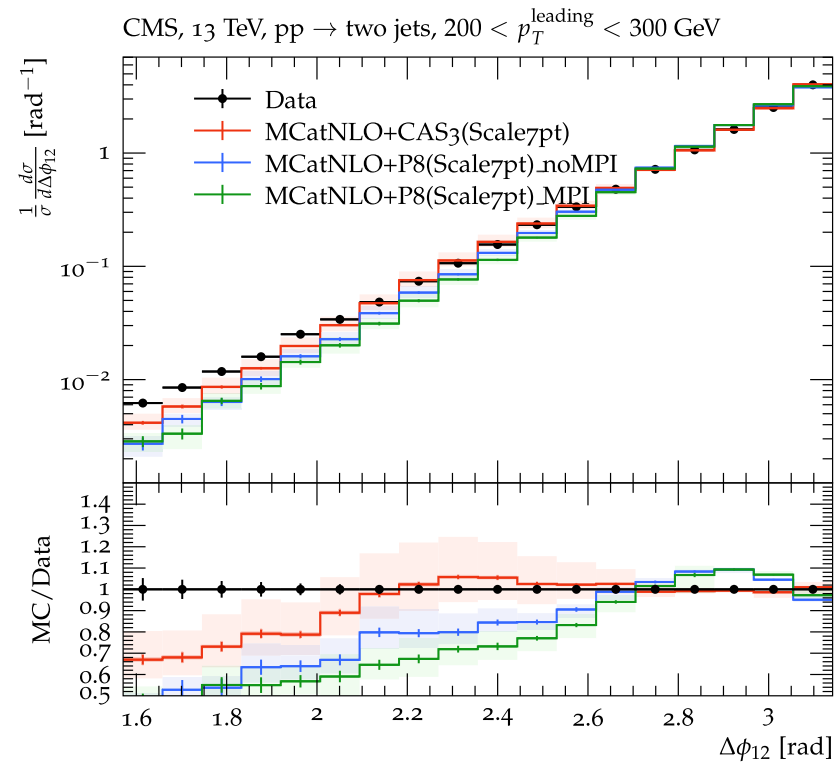

Fig. 8 Azimuthal correlation $\Delta \phi_{12}$ over a wide range and (left) in the back-to-back region (right) for $p_{\mathrm{T}}^{\text {leading }}>200 \mathrm{GeV}$ compared with pre-

ment and the prediction is observed for smaller $\Delta \phi_{12}$ which is due to missing higher order corrections in the matrix element calculation. Even at high $p_{\mathrm{T}}^{\text {leading }}>1000 \mathrm{GeV}$ the prediction is in agreement with the measurements (within uncertainties), while only in the highest $\Delta \phi_{12}$ bin $\left(\Delta \phi_{12}>179^{\circ}\right)$ a deviation of about $10 \%$ is observed.

In Figs. 6 and 7, the predictions using PB-NLO-2018Set 1 are compared with those from PB-NLO-2018-Set 2 and with the measurements. The difference between Set 1 and Set 2 becomes significant in the back-to-back region, which is sensitive to the low $k_{\mathrm{T}}$-region of the TMD. As already observed in the case of Z-boson production in Ref. [42], Set 2 with the transverse momentum as a scale for $\alpha_{\mathrm{s}}$, which is required from angular ordering conditions, allows a much better description of the measurement. It has been explicitly checked that the choice of the collinear parton density function (in contrast to the choice of the TMD densities) does not matter for the $\Delta \phi_{12}$ distributions, since they are normalized. The region of low $\Delta \phi_{12}$ in Figs. 4 and 6 is not well described with an NLO dijet matrix element calculation supplemented with TMD densities and TMD parton shower because in the low $\Delta \phi_{12}$ region higher-order hard emissions play a significant role. It has been shown in [67] that the inclusion of higher order matrix elements with the new TMD merging method of Ref. [44] leads to a very good description of the low $\Delta \phi_{12}$ region.

In Fig. 8 predictions obtained with MCatNLO+PyTHIA8 are compared with MCatNLO+CAS3. In the calculation of MCatNLO+PYTHIA8, the PYTHIA8 subtraction terms are

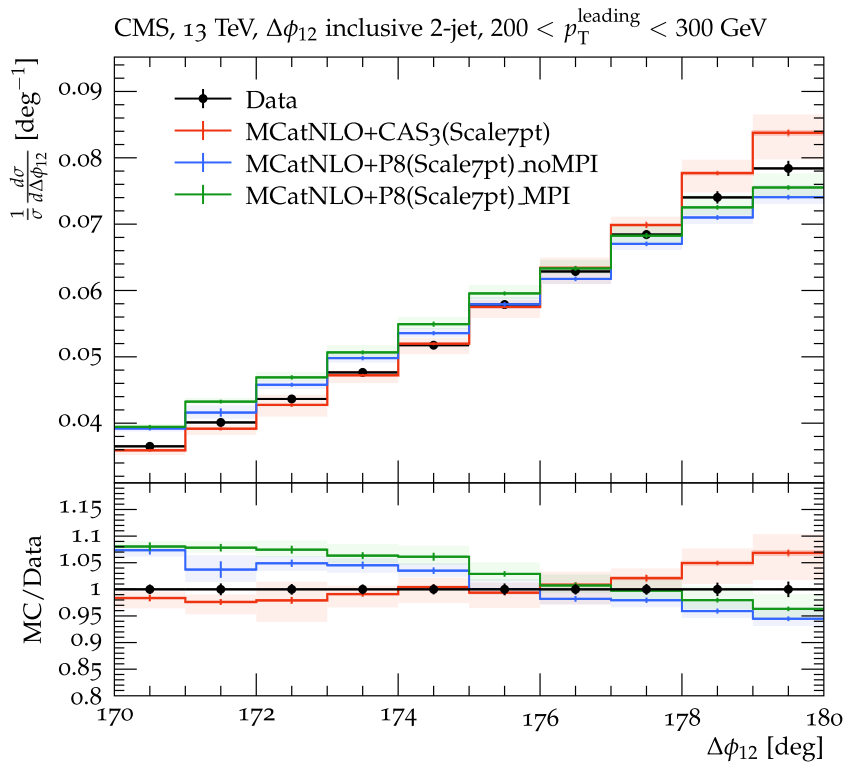

dictions from MCatNLO+PYTHIA8 and MCatNLO+CAS3. The uncertainties in the MCatNLO+PYTHIA8 calculation are obtained from scale and associated shower variations, as described in the main text

used and the NNPDF3.0 [68] parton density and tune CUETP8M1 [69] are applied. The uncertainties of the PYTHIA prediction are derived by combining the fixed-order scale variation from MCatNLO with renormalization scale variations in the parton shower. We use the method of [70] together with the guidelines of [71] to obtain consistent scale variations where possible. In particular, this means that the renormalization scale variation at fixed order and in the parton shower are fully correlated. ${ }^{1}$ The factorization scale variation is only applied at fixed order, as argued in [71]. We observe a significant dependence on the matching scale $\mu_{m}$, the details of matching in case of dijets needs further investigation.

Shown in Fig. 8 is also the contribution from multiparton interactions, which is very small for jets with $p_{\mathrm{T}}^{\text {leading }}>$ $200 \mathrm{GeV}$. The prediction obtained with MCatNLO+PYTHIA8 is in all $\Delta \phi_{12}$ regions different from the measurement and MCatNLO+CAS3, illustrating the role of the treatment of parton showers.

In conclusion, the predictions of MCatNLO+CAS3 are in reasonable agreement with the measurements in the larger $\Delta \phi_{12}$ regions, where the contribution from higher order matrix elements is small. In the back-to-back region $\left(\Delta \phi_{12} \rightarrow \pi\right)$ the predictions obtained with PB-TMDs and

\footnotetext{
1 This also ensures that for fixed-order-dominated observables, the cancellation between the expansion of the shower and the subtraction in MC@NLO also occurs for non-central renormalization scales without significant deformation of the - there fully appropriate - fixed-order uncertainties.
} 
parton shower are in good agreement with the measurement. The uncertainties of the predictions are dominated by the scale uncertainties of the matrix element calculations, while the PB-TMD and TMD shower uncertainties are very small, as they are directly coming from the uncertainties of the PBTMDs. No uncertainties, in addition to those from the PBTMD, come from the PB-TMD parton shower.

\section{Conclusion}

We have investigated the azimuthal correlation of high transverse momentum jets in pp collisions at $\sqrt{s}=13 \mathrm{TeV}$ by applying PB-TMD distributions to NLO calculations via MCatNLO. We use the same PB-TMDs and MCatNLO calculations as we have used for $\mathrm{Z}$-production at LHC energies in Ref. [42]. A very good description of the cross section as a function of $\Delta \phi_{12}$ is observed. In the back-to-back region of $\Delta \phi_{12} \rightarrow \pi$ a very good agreement is observed with PBTMD Set 2 distributions [51] while significant differences are obtained with PB-TMD Set 1 distributions, which use the evolution scale as an argument in $\alpha_{\mathrm{s}}$. This observation confirms the importance of consistently handling the softgluon coupling in angular ordered parton evolution.

The uncertainties of the predictions are dominated by the scale uncertainties of the matrix element, while uncertainties coming from the PB-TMDs and the corresponding PB-TMD shower are very small. No other uncertainties, in addition to those of the PB-TMD, come from the PB-TMD shower, since it is directly correlated with the PB-TMD density.

We have also investigated predictions using MCatNLO with PYTHIA8 to illustrate the importance of details of the parton shower.

Acknowledgements This is based in part on studies during the "Virtual Monte Carlo school - PB TMDs with CASCADE3" [72] held from 8.12. November 2021 at DESY, Hamburg. We are grateful to Olivier Mattelaer from the MADGRAPH5_AMC@NLO team for discussions, help and support with the LHE option for fixed NLO calculations in MCatNLO. STM thanks the Humboldt Foundation for the Georg Forster research fellowship and gratefully acknowledges support from IPM. D. E. Martins is supported by CNPQ-Brazil, process 164609/2020-2.

Data Availability Statement This manuscript has no associated data or the data will not be deposited. [Authors' comment: There are no data to be deposited: the measurements are already on HEPdata.]

Open Access This article is licensed under a Creative Commons Attribution 4.0 International License, which permits use, sharing, adaptation, distribution and reproduction in any medium or format, as long as you give appropriate credit to the original author(s) and the source, provide a link to the Creative Commons licence, and indicate if changes were made. The images or other third party material in this article are included in the article's Creative Commons licence, unless indicated otherwise in a credit line to the material. If material is not included in the article's Creative Commons licence and your intended use is not permitted by statutory regulation or exceeds the permit- ted use, you will need to obtain permission directly from the copyright holder. To view a copy of this licence, visit http://creativecomm ons.org/licenses/by/4.0/.

Funded by SCOAP 3 .

\section{References}

1. ATLAS Collaboration, Measurement of the inclusive jet cross section in pp collisions at $\mathrm{sqrt}(\mathrm{s})=2.76 \mathrm{TeV}$ and comparison to the inclusive jet cross section at sqrt(s) $=7 \mathrm{TeV}$ using the ATLAS detector. Eur. Phys. J. C 73(8), 2509 (2013). arXiv:1304.4739

2. CMS Collaboration, Measurement of the inclusive jet cross section in pp collisions at $\sqrt{s}=2.76 \mathrm{TeV}$. Eur. Phys. J. C 76(5), 265 (2016). arXiv: 1512.06212

3. CMS Collaboration, Measurements of differential jet cross sections in proton-proton collisions at $\sqrt{s}=7 \mathrm{TeV}$ with the CMS detector. Phys. Rev. D 87(11), 112002 (2013). arXiv:1212.6660. [Erratum: Phys. Rev. D 87, 119902 (2013)]

4. CMS Collaboration, Measurement of the ratio of inclusive jet cross sections using the anti- $k_{T}$ algorithm with radius parameters $\mathrm{R}=0.5$ and 0.7 in pp collisions at $\sqrt{s}=7 \mathrm{TeV}$. Phys. Rev. D 90, 072006 (2014). arXiv:1406.0324

5. ATLAS Collaboration, Measurement of the inclusive jet crosssections in proton-proton collisions at $\sqrt{s}=8 \mathrm{TeV}$ with the ATLAS detector. JHEP 09, 020 (2017). arXiv: 1706.03192

6. CMS Collaboration, Measurement and QCD analysis of doubledifferential inclusive jet cross sections in pp collisions at $\sqrt{s}=$ $8 \mathrm{TeV}$ and cross section ratios to 2.76 and $7 \mathrm{TeV}$. JHEP 03, 156 (2017). arXiv: 1609.05331

7. ATLAS Collaboration, Measurement of inclusive jet and dijet cross-sections in proton-proton collisions at $\sqrt{s}=13 \mathrm{TeV}$ with the ATLAS detector. JHEP 05, 195 (2018). arXiv:1711.02692

8. CMS Collaboration, Measurement of the double-differential inclusive jet cross section in proton-proton collisions at $\sqrt{s}=13 \mathrm{TeV}$. Eur. Phys. J. C 76, 451 (2016). arXiv: 1605.04436

9. CMS Collaboration, Dependence of inclusive jet production on the anti- $k_{\mathrm{T}}$ distance parameter in pp collisions at $\sqrt{s}=13 \mathrm{TeV}$. JHEP 12, 082 (2020). arXiv:2005.05159

10. ALICE Collaboration, Measurements of inclusive jet spectra in $\mathrm{pp}$ and central $\mathrm{Pb}-\mathrm{Pb}$ collisions at $\sqrt{s_{\mathrm{NN}}}=5.02 \mathrm{TeV}$. Phys. Rev. C 101, 034911 (2020). arXiv:1909.09718

11. ATLAS Collaboration, Measurement of inclusive jet and dijet production in pp collisions at sqrt(s) $=7 \mathrm{TeV}$ using the ATLAS detector. Phys. Rev. D 86, 014022 (2012). arXiv:1112.6297

12. ATLAS Collaboration, Measurement of inclusive jet and dijet cross sections in proton-proton collisions at $7 \mathrm{TeV}$ centre-of-mass energy with the ATLAS detector. Eur. Phys. J. C 71, 1512 (2011). arXiv: 1009.5908

13. S. Alioli et al., Jet pair production in POWHEG. JHEP 04, 081 (2011). arXiv: 1012.3380

14. Z. Nagy, Three jet cross-sections in hadron hadron collisions at next-to-leading order. Phys. Rev. Lett. 88, 122003 (2002). arXiv:hep-ph/0110315

15. W.T. Giele, E.W.N. Glover, D.A. Kosower, The two-jet differential cross section at $\mathcal{O}\left(\alpha_{s}^{3}\right)$ in hadron collisions. Phys. Rev. Lett. 73, 2019-2022 (1994). arXiv:hep-ph/9403347

16. S.D. Ellis, Z. Kunszt, D.E. Soper, Two jet production in hadron collisions at order $\alpha_{s}^{3}$ in QCD. Phys. Rev. Lett. 69, 1496 (1992)

17. M. Czakon, A. van Hameren, A. Mitov, R. Poncelet, Single-jet inclusive rates with exact color at $\mathcal{O}\left(\alpha_{s}^{4}\right)$. JHEP 10, 262 (2019). arXiv:1907.12911

18. A. Gehrmann-De Ridder et al., Triple differential dijet cross section at the LHC. Phys. Rev. Lett. 123(10), 102001 (2019). arXiv:1905.09047 
19. J. Currie, E.W.N. Glover, J. Pires, Next-to-next-to leading order QCD predictions for single jet inclusive production at the LHC. Phys. Rev. Lett. 118(7), 072002 (2017). arXiv:1611.01460

20. J. Currie et al., Single jet inclusive production for the individual jet $p_{\mathrm{T}}$ scale choice at the LHC. Acta Phys. Pol. B 48, 955-967 (2017). arXiv: 1704.00923

21. D0 Collaboration, Measurement of dijet azimuthal decorrelations at central rapidities in $p \bar{p}$ collisions at $\sqrt{s}=1.96 \mathrm{TeV}$. Phys. Rev. Lett. 94, 221801 (2005). arXiv:hep-ex/0409040

22. D0 Collaboration, Measurement of the combined rapidity and $p_{T}$ dependence of dijet azimuthal decorrelations in $p \bar{p}$ collisions at $\sqrt{s}=1.96 \mathrm{TeV}$. Phys. Lett. B 721, 212-219 (2013). arXiv: 1212.1842

23. ATLAS Collaboration, Measurement of dijet azimuthal decorrelations in pp collisions at sqrt(s) $=7 \mathrm{TeV}$. Phys. Rev. Lett. 106, 172002 (2011). arXiv:1102.2696

24. CMS Collaboration, Dijet azimuthal decorrelations in pp collisions at sqrt(s) $=7$ TeV. Phys. Rev. Lett. 106, 122003 (2011). arXiv: 1101.5029

25. CMS Collaboration, Measurement of dijet azimuthal decorrelation in pp collisions at $\sqrt{s}=8 \mathrm{TeV}$. Eur. Phys. J. C 76, 536 (2016). arXiv: 1602.04384

26. CMS Collaboration, Azimuthal correlations for inclusive 2-jet, 3jet, and 4-jet events in pp collisions at $\sqrt{s}=13 \mathrm{TeV}$. Eur. Phys. J. C 78, 566 (2018). arXiv:1712.05471

27. CMS Collaboration, Azimuthal separation in nearly back-to-back jet topologies in inclusive 2- and 3-jet events in pp collisions at $\sqrt{s}=13$ TeV. Eur. Phys. J. C 79, 773 (2019). arXiv: 1902.04374

28. R. Angeles-Martinez et al., Transverse momentum dependent (TMD) parton distribution functions: status and prospects. Acta Phys. Pol. B 46(12), 2501 (2015). arXiv:1507.05267

29. S. Dooling, P. Gunnellini, F. Hautmann, H. Jung, Longitudinal momentum shifts, showering and nonperturbative corrections in matched NLO-shower event generators. Phys. Rev. D 87, 094009 (2013). arXiv:1212.6164

30. F. Hautmann, H. Jung, Collinearity approximations and kinematic shifts in partonic shower algorithms. Eur. Phys. J. C 72, 2254 (2012). arXiv:1209.6549

31. A. Banfi, M. Dasgupta, Y. Delenda, Azimuthal decorrelations between QCD jets at all orders. Phys. Lett. B 665, 86-91 (2008). arXiv:0804.3786

32. P. Sun, C.P. Yuan, F. Yuan, Soft gluon resummations in dijet azimuthal angular correlations in hadronic collisions. Phys. Rev. Lett. 113(23), 232001 (2014). arXiv:1405.1105

33. P. Sun, C.P. Yuan, F. Yuan, Transverse momentum resummation for dijet correlation in hadronic collisions. Phys. Rev. D 92(9), 094007 (2015). arXiv:1506.06170

34. Y. Hatta, B.-W. Xiao, F. Yuan, J. Zhou, Azimuthal angular asymmetry of soft gluon radiation in jet production. Phys. Rev. D 104(5), 054037 (2021). arXiv:2106.05307

35. Y. Hatta, B.-W. Xiao, F. Yuan, J. Zhou, Anisotropy in dijet production in exclusive and inclusive processes. Phys. Rev. Lett. 126(14), 142001 (2021). arXiv:2010.10774

36. J. Collins, J.-W. Qiu, $k_{T}$ factorization is violated in production of high-transverse-momentum particles in hadron-hadron collisions. Phys. Rev. D 75, 114014 (2007). arXiv:0705.2141

37. W. Vogelsang, F. Yuan, Hadronic dijet imbalance and transversemomentum dependent parton distributions. Phys. Rev. D 76, 094013 (2007). arXiv:0708.4398

38. T.C. Rogers, P.J. Mulders, No generalized TMD-factorization in hadro-production of high transverse momentum hadrons. Phys. Rev. D 81, 094006 (2010). arXiv:1001.2977

39. F. Hautmann et al., Soft-gluon resolution scale in QCD evolution equations. Phys. Lett. B 772, 446 (2017). arXiv:1704.01757
40. F. Hautmann et al., Collinear and TMD quark and gluon densities from Parton Branching solution of QCD evolution equations. JHEP 01, 070 (2018). arXiv: 1708.03279

41. S. Drell, T.-M. Yan, Massive lepton pair production in hadronhadron collisions at high-energies. Phys. Rev. Lett. 25, 316-320 (1970)

42. A. Bermudez Martinez et al., Production of Z-bosons in the parton branching method. Phys. Rev. D 100, 074027 (2019). arXiv: 1906.00919

43. A. Bermudez Martinez et al., The transverse momentum spectrum of low mass Drell-Yan production at next-to-leading order in the parton branching method. Eur. Phys. J. C 80, 598 (2020). arXiv:2001.06488

44. A. Bermudez Martinez, F. Hautmann, M.L. Mangano, TMD evolution and multi-jet merging. Phys. Lett. B 822, 136700 (2021). arXiv:2107.01224

45. S.P. Baranov et al., Discriminating the heavy jet production mechanisms in associated $Z+$ heavy flavor events at the LHC. arXiv:2111.04521

46. F. Hautmann, H. Jung, Angular correlations in multi-jet final states from kt-dependent parton showers. JHEP 10, 113 (2008). arXiv:0805.1049

47. S. Dooling, F. Hautmann, H. Jung, Hadroproduction of electroweak gauge boson plus jets and TMD parton density functions. Phys. Lett. B 736, 293 (2014). arXiv:1406.2994

48. M. Bury et al., Calculations with off-shell matrix elements, TMD parton densities and TMD parton showers. Eur. Phys. J. C 78, 137 (2018). arXiv: 1712.05932

49. J. Alwall et al., The automated computation of tree-level and next-to-leading order differential cross sections, and their matching to parton shower simulations. JHEP 1407, 079 (2014). arXiv: 1405.0301

50. S. Baranov et al., CASCADE3 A Monte Carlo event generator based on TMDs. Eur. Phys. J. C 81, 425 (2021). arXiv:2101.10221

51. A. BermudezMartinez et al., Collinear and TMD parton densities from fits to precision DIS measurements in the parton branching method. Phys. Rev. D 99, 074008 (2019). arXiv:1804.11152

52. ZEUS, H1 Collaboration, Combination of measurements of inclusive deep inelastic $e^{ \pm} p$ scattering cross sections and QCD analysis of HERA data. Eur. Phys. J. C 75, 580 (2015). arXiv:1506.06042

53. N.A. Abdulov et al., TMDlib2 and TMDplotter: a platform for 3D hadron structure studies. Eur. Phys. J. C 81, 752 (2021). arXiv:2103.09741

54. F. Hautmann et al., TMDlib and TMDplotter: library and plotting tools for transverse-momentum-dependent parton distributions. Eur. Phys. J. C 74(12), 3220 (2014). arXiv:1408.3015

55. A. Bassetto, M. Ciafaloni, G. Marchesini, Jet structure and infrared sensitive quantities in perturbative QCD. Phys. Rep. 100, 201-272 (1983)

56. Y.L. Dokshitzer, V.A. Khoze, S.I. Troian, A.H. Mueller, QCD coherence in high-energy reactions. Rev. Mod. Phys. 60, 373 (1988)

57. S. Catani, B.R. Webber, G. Marchesini, QCD coherent branching and semiinclusive processes at large x. Nucl. Phys. B 349, 635-654 (1991)

58. F. Hautmann, L. Keersmaekers, A. Lelek, A.M. Van Kampen, Dynamical resolution scale in transverse momentum distributions at the LHC. Nucl. Phys. B 949, 114795 (2019). arXiv:1908.08524

59. A. Buckley et al., LHAPDF6: parton density access in the LHC precision era. Eur. Phys. J. C 75, 132 (2015). arXiv:1412.7420

60. J. Alwall et al., A standard format for Les Houches event files. Comput. Phys. Commun. 176, 300 (2007). arXiv:hep-ph/0609017

61. A. Buckley et al., Rivet user manual. Comput. Phys. Commun. 184, 2803-2819 (2013). arXiv:1003.0694

62. G. Corcella et al., HERWIG 6.5 release note. arXiv:hep-ph/0210213 
63. G. Marchesini et al., HERWIG: a Monte Carlo event generator for simulating hadron emission reactions with interfering gluons. Version 5.1-April 1991. Comput. Phys. Commun. 67, 465-508 (1992)

64. T. Sjöstrand, S. Mrenna, P. Skands, PYTHIA 6.4 physics and manual. JHEP 05, 026 (2006). arXiv:hep-ph/0603175

65. M. Cacciari, G.P. Salam, G. Soyez, The anti- $k_{t}$ jet clustering algorithm. JHEP 04, 063 (2008). arXiv:0802.1189

66. M. Cacciari, G.P. Salam, G. Soyez, FastJet user manual. Eur. Phys. J. C 72, 1896 (2012). arXiv:1111.6097

67. A. Bermudez Martinez et al., Jet production and TMD evolution. Presented at the workshop on Resummation, Evolution, Factorization (REF2021), DESY (2021)

68. NNPDF Collaboration, Parton distributions for the LHC Run II. JHEP 04, 040 (2015). arXiv: 1410.8849

69. CMS Collaboration, Event generator tunes obtained from underlying event and multiparton scattering measurements. Eur. Phys. J. C 76, 155 (2016). arXiv: 1512.00815
70. S. Mrenna, P. Skands, Automated parton-shower variations in Pythia 8. Phys. Rev. D 94(7), 074005 (2016). arXiv:1605.08352

71. L. Gellersen, S. Prestel, Scale and scheme variations in unitarized NLO merging. Phys. Rev. D 101(11), 114007 (2020). arXiv:2001.10746

72. A. Bermudez Martinez, H. Jung, S. Taheri Monfared, Q. Wang, Virtual Monte Carlo school-PB TMDs with CASCADE (2021). https://indico.desy.de/event/31877/ 